\title{
DYNAMIC-FUZZY CONCEPTS
}

\author{
Junquan $\mathrm{Li}^{1}$ Andrzej Buller ${ }^{2}$ Yixin Yin ${ }^{3}$ \\ 1. 3School of Information Engineering, University of Science and Technology \\ Beijing, Haidian Zone, Beijing 100083, China, \\ 2 ATR International Network Informatics Laboratories, Kyoto,JapanE-mail: \\ tg097rr@163.com;AndrzejBuller@atr.jp; yyx@ies.ustb.edu.cn
}

Abstract: This paper presents dynamic-fuzzy logic (DFL) and its usefulness in cognitive/affective modeling. Freud noted that hate changes into love and love into hate even without any alteration of the object. Vallacher and Nowak confirmed experimentaily that people might change their judgment even in absence of new data of the object. Related mental phenomena can be modeled using fuzzy sets, provided that constant membership values are replaced with dynamic ones. As for definition of membership, instead of a real numbers in the interval $[0,1]$ Buller proposed a function mapping time onto this interval. This way a membership becomes a dynamic membership and fuzzy sets become dynamic-fuzzy. A cellular inference engine, called Working Memory, was used for dynamic-fuzzy calculus. Fuzziness of input data was represented there as streams of contradictory statements called memes. When two or more memes met in a cell, they may elastically collide, annihilate, or exchange/invert some of their codes.

Keywords: dynamic fuzziness, working memory, social judgment

\section{INTRODUCTION}

As Sigmund Freud noted, in a number of circumstances hate changes into love and love into hate. The changes are purely internal and an alteration of the object plays no part in them [7]. Robin Vallacher and Andrzej Nowak, based on experiments with human subjects, confirmed that when judging a perceived person or social situation people sometimes switch from highly positive feelings to highly negative ones even in the absence of new data about the object of interest [10]. Andrzej Buller [4] considered the question: 'Can we model this mental phenomenon using 
fuzzy sets?' and proposed to replace constant values of memberships to fuzzy sets with dynamic ones, or, in other words, instead of a real number in the interval $[0 ; 1]$, to employ a function mapping time onto the interval. Fuzzy logic dealing with dynamic memberships to sets is called Dynamic-Fuzzy Logic (DFL).

The following example from the domain of social psychology (taken from [4]) explains the idea of dynamic membership. Let us consider a situation wherein two subjects $S^{\prime}$ and $S^{\prime \prime}$ one-day fall in love with the same person P. Among a number of possible plots of the subjects' feelings towards $P$, let us assume that the feelings of $S^{\prime}$ start.from extreme love and then a linear decay of the fascination takes place towards complete indifference after three weeks, while $S$ "feels constant and extreme love that after two weeks turns without any external reasons into a deep aversion. Let us try to describe the feelings of the subjects in terms of classic fuzzy sets. We could provide information on the subjects' feelings in a particular moment in time or an average value for a period of interest (e.g. three weeks). Let us introduce fuzzy sets $\mathrm{H}^{\prime}, \mathrm{H}^{\prime \prime}, \mathrm{L}^{\prime}$, and $\mathrm{L}^{\prime \prime}$, which contain people hated by $\mathrm{S}^{\prime}$, people hated by $S^{\prime \prime}$, people loved by $S^{\prime}$, and people loved by $S^{\prime \prime}$, respectively. For the last day of the period of interest, the fuzzy-set-theoretic membership values can be provided as, say: $\mu_{\mathrm{H}^{\prime}}(P)=0, \mu_{\mathrm{H}^{\prime \prime}}(P)=0, \mu_{\mathrm{L}^{\prime}}(P)=1, \mu_{\mathrm{L}^{\prime \prime}}(P)=0$. Average values covering the full period of interest (three weeks) could be: $\mu_{\mathrm{H}^{\prime}}(P)=0, \mu_{\mathrm{H}^{\prime \prime}}(P)=0.33, \mu_{\mathrm{L}}(P)=0.5, \mu_{\mathrm{L}^{\prime \prime}}(P)=0.67$. Unfortunately, the provided figures are not too informative. In both cases the most essential knowledge about the subjects' mental states is lost. Hence, the idea of a replacement of constant membership values $\mu_{\mathrm{H}^{\prime}}(P), \mu_{\mathrm{H}^{\prime \prime}}(P), \ldots$ with functions of time, i.e. $\mu_{\mathrm{H}^{\prime}}(P, \mathrm{t}), \mu_{\mathrm{H}^{\prime \prime}}(P, \mathrm{t}), \ldots$, respectively are suggested. These functions are dynamic memberships.

It can be noted, that if for a period of interest, the values of the dynamic membership functions remained constant (or almost constant), the sets would be subject to classic fuzzy calculus. DFL was introduced to handle dynamic memberships. It is assumed in DFL that based on a set of functions describing dynamic memberships of objects $e_{1}, e_{2}, \ldots$ to each of dynamic fuzzy sets $D^{\prime}$, $D^{\prime \prime}, \ldots$, where plots of some of the functions are unknown, an updated set of the functions (i.e. a set containing the missing definitions) is to be produced [4]. A cellular inference engine, called Working Memory, was used for dynamic-fuzzy calculus. Fuzziness of input data was represented there as streams of contradictory statements called memes. When two or more memes met in a cell, an interaction took place [3][5][6].

\section{DYNAMIC FUZZY CALCULUS}

Buller et al. investigated several models of cellular- automata-based Working Memory [2][3][5][6]. In all of them fuzziness of input data was represented as streams of contradictory statements introduced in [1]. The following example (taken 
from [4]) explains the idea of dynamic-fuzzy calculus. Let us consider milk as an example of an object of interest $\theta$. Let us assume that $\theta$ is recognized as suitable for baby feeding if it is warm. When, based on perceived data, membership of $\theta$ to the classic fuzzy set $W$, where $W$ denotes "warm drinks", is $\mu_{W}(\theta)$, a stream of identical copies of the statement " $\theta \in W$ ", as well as a stream of identical copies of the statement " $\theta \in \neg W$ " are being directed to Working Memory. The statements have been christened memes. Notation " $W$ " is for "drinks that are not warm". The existence of the two contradictory meme streams represent a mother's uncertainty whether the milk is warm enough. Let us assume that the densities of the streams are $\sigma_{t}\left(" \theta \in W^{\prime \prime}\right)=\sigma\left(" \theta \in W^{\prime \prime}\right)=$ const. and $\sigma_{t}\left(\right.$ (" $\left.\theta \in \neg W^{\prime \prime}\right)=\sigma(" \theta \in \neg W ")=$ const., respectively, and such that $\mu_{W}(\theta)=\sigma$ " $\left.\theta \in W "\right) /(\sigma(" \theta \in W ")+\sigma(" \theta \in$ $\neg W$ ')). The streams cause the Working Memory to become a space populated by a "society" of contradictory memes. Let us assume that a stream of identical copies of the meme " $W \subset P$ ", where $P$ denotes the set of "drinks proper for baby feeding", is also directed to Working Memory. Dynamic fuzzy inferencing consists in interactions between memes populating Working Memory and production of output streams of memes " $\theta \in P$ " and " $\theta \in \neg P$ ". The deduced $\mu_{P}(\theta, t)$ equals $\sigma_{t}$ (" $\theta \in$ $P ") /\left(\sigma_{t}\left(" \theta \in P\right.\right.$ ") $+\sigma_{t}(" \theta \in \neg P$ "). The above example becomes more interesting when the streams of memes " $\theta \in H$ ", " $\theta \in \neg H$ " and " $\neg H \subset P$ ", where $H$ is a set of "hot drinks", are also involved.

Since Working Memory is implemented as a cellular automaton, memes represented as binary words wander all over the cellular space. When two or more memes meet in a cell, an interaction takes place. Via trial and error, a set of meme interactions causing a psychologically plausible dynamics of simulated mental states has been obtained. The interactions are:

(i) elastic collision between unrelated memes;

(ii) annihilation of contradictory memes,

e.g. " $\theta \in W$ ", " $\theta \in \neg W$ " $\rightarrow$ null;

(iii) positive cross-over,

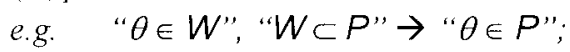

(iv) negative cross-over,

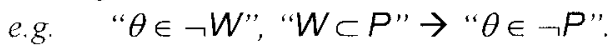

Simulation experiments confirmed that even in case of constant streams of input memes, the densities of the resulting streams may vary in time. This phenomenon takes place especially when the populations of resulting contradictory memes have an equal or almost equal "birth rate". Instead of chaotic oscillations or a convergence to a "level of balance", a given population of memes may enjoy its quick victory (i.e. dominate Working Memory) and, after a period of time, suddenly lose to the population of contradictory memes. This dramatic change may refer to a case when a mother recognizes milk as warm enough for her baby, and when going to feed it, changes her mind and decides to warm the milk a little more. 
Similar irregular oscillations of meme population was observed in a model of a subject's hesitation how to react to date proposal (Fig. 1), as well as in a model of a robot's hesitation whether to go to recharge batteries or to continue playing with an attractive toy [9]. Recently leaky neurons are investigates as units facilitating dynamics-fuzzy calculus applied to robotics [8].

\section{CONCLUDING REMARKS}

Dynamic membership offered by Dynamic- Fuzzy Logic (DFL) is a convenient concept to be used for modeling high-level mental functions including social judgment. On the other hand, the cellular Working Memory proposed in DFL-related papers does not seem to be the best way to process dynamic-fuzzy concepts because of big computational power required. Hence, further research on DFL should seek for simple neural networks capable of processing dynamic-fuzzy data.

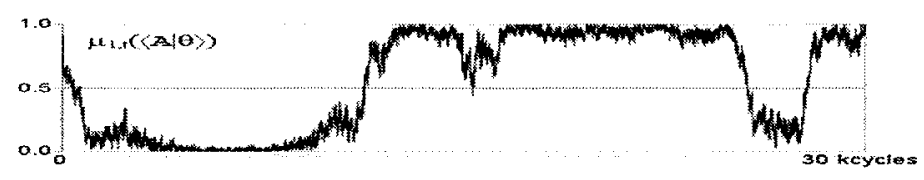

Fig. 1. Dynamics of meme populations in cellular Working Memory. Oscillating feelings of a simulated subject represented as dynamic-fuzzy membership of an object of interest $(\theta)$ to a dynamic fuzzy set $\boldsymbol{A}$ (adopted from [3]).

\section{Acknowledgements}

This research was supported by NSFC, China (Grant No.60374032). The authors thank Dr. Andrzej Buller and ATR.

\section{REFERENCES}

[1] A. Buller, Fuzzy Inferencing: A Novel, Massively Parallel Approach, Technical Report TR-95-027, Intnl. Computer Sci. Institute, Berkeley, CA, 1995.

[2] A. Buller, "Dynamic Fuzzy Sets for Cognitive Modeling", Proceedings, AROB 6th '01, Tokyo, 15-17 Jan., pp. 150-151, 2001.

[3] A. Buller, "Dynamic Fuzziness", Proceedings, $7^{\text {th }}$ Pacific Rim Intnl Conf. on Artificial Intelligence (PRICAI-02), Tokyo, pp. 90-96, 18-22 August, 2002. 
[4] A. Buller, "Fuzzy Sets with Dynamic Memberships", FSKD'02, 18-22 Nov., Singapore, pp. 564-565, 2002.

[5] A. Buller \& K. Shimohara, "Decision Making as a Debate in the Society of Memes in a Neural Working Memory", J. of 3D Forum, 13 (3), pp. 77-82, 1999.

[6] A. Buller \& K. Shimohara, "On the dynamics of judgment: does the butterfly effect take place in human working memory?", Artificial Life and Robotics, Vol. 5, No. 2, pp. 88-92, 2001.

[7] S. Freud, The Ego and the Id, W.W. Norton \& Company, New York, pp. 41-42, $1923 / 1990$.

[8] J. Li, A. Buller \& J. Liu, "Robot's behavior driven by internal tensions regulated by pulsed para-neural networks", AROB 10 ${ }^{\text {th }}$ 05, Beppu, 4-6 Feb., 2005.

[9] J. Liu \& A. Buller, "Tensions and conflicts: Toward a pleasure-seeking artifact", Proceedings, 5th IFAC Symposium on Intelligent Autonomous Vehicles (IAV'2004), 5-7 July, Lisboa, 2004.

[10] R.A. Vallacher \& A. Nowak, "The Stream of Social Judgment", In: R.A. Vallacher \& A. Nowak (Eds.) Dynamical Systems in Social Psychology, Academic Press: San Diego, pp. 251-277, 1994. 